For nal ver i fi cat i on of dynamical I y reconf i gur abl e syst ens

\begin{tabular}{|l|l|}
\hline 著者 & $\begin{array}{l}\text { Yanase Ryo, Sakai Tat sunor i , Sakai Nakoto, } \\
\text { Yamane Sat oshi }\end{array}$ \\
\hline $\begin{array}{l}\text { j our nal or } \\
\text { publ i cat i on t i t l e }\end{array}$ & $\begin{array}{l}\text { 2015 I EEE 4t h G obal Conf er ence on Consumer } \\
\text { El ect r oni cs }\end{array}$ \\
\hline vol une & GCCE 2015 \\
\hline number & 7398597 \\
\hline page $r$ ange & $71-75$ \\
\hline year & $2016-02-03$ \\
\hline URL & ht t p: //hdl . handl e. net /2297/45586 \\
\hline
\end{tabular}




\title{
Formal Verification of Dynamically Reconfigurable Systems
}

\author{
Ryo Yanase *, Tatsunori Sakai *, Makoto Sakai * and Satoshi Yamane ${ }^{\dagger}$ \\ * Graduate School of Natural Science and Technology \\ Kanazawa University, Kakuma-machi 920-1192 \\ Email: ryanase@csl.ec.t.kanazawa-u.ac.jp \\ $\dagger$ Institute of Science and Engineering \\ Kanazawa University, Kakuma-machi 920-1192 \\ Email: syamane@is.t.kanazawa-u.ac.jp
}

\begin{abstract}
A dynamically reconfigurable system can perform complicated operations with dynamically changing the configuration. For ensuring the safety of the system, a model checking is one of the efficient formal approach. In our work, we define the specification language of a dynamically reconfigurable system and propose the model checking algorithm of verifying safety properties.
\end{abstract}

\section{INTRODUCTION}

If a system changes its configuration during the operation, we call it dynamically reconfigurable system. The feature of a dynamically reconfigurable system is appeared in many realtime systems (e.g., embedded systems, networks, etc.). Since the operation of a such system is generally complex, it is difficult to guarantee that the system is safe. Model checking is a formal method for verification, and it is one of the effective approach. In our work, we define the specification language dynamic linear hybrid automaton (DLHA) of dynamically reconfigurable systems and propose an approach to the model checking of safety properties.

\section{RELATED WORKS}

Linear hybrid automaton provides continuous and discrete operations but cannot describe the dynamic change of the configuration[1]. Therefore, the system is modeled as a static system[2]. Dynamic Input/Output automaton describes asynchronous concurrent systems with FIfO channels and also provides the dynamic change as creation and destruction of automata[3]. However, this language doesn't provide continuous transition and cannot describe a real-time system. Hierarchical linear hybrid automaton is based on concepts of object-orientation[4]. For this language, given a large scale system, the specification and the verification method tend to be complex. H. Nakano and others have developed a dynamically configurable processor LSI that the circuit configuration is changed by a single clock[5]. In our work, we assume that a dynamically reconfigurable system can change its configuration by a single clock.

\section{SPECIFICATION LANGUAGE}

We propose a specification language Dynamic Linear Hybrid Automaton (DLHA). A DLHA is a hybrid automaton extended by creation/destruction of automata and queue operations.

\section{A. Dynamic Linear Hybrid Automaton}

A dynamic linear hybrid automaton(DLHA) is a linear hybrid automaton extended with special actions that are labels representing the types of transitions.

1) Syntax of a DLHA: A DLHA is a tuple consisting of the following components:

- $\quad$ A finite set $L$ of locations

- $\quad$ A finite set $V$ of variables

- A function Inv that assigns a constraint to each location: A constraint $\phi$ on $V$ is defined by

$$
\phi::=\text { true }|x \sim e| x-y \sim e \mid \phi_{1} \wedge \phi_{2}
$$

where $x, y \in V, e \in \mathbb{Q}, \phi_{1}$ and $\phi_{2}$ are constraints on $V$, and $\sim \in\{=,<,>, \leq, \geq\}$. $\Phi(V)$ denotes a set of all constraints on $V$. For a location $l \in L$, a constraint $\operatorname{Inv}(l)$ is called invariant of $l$.

- A function Flow that assigns a flow condition to each location: Let $V=\left\{x_{1}, \ldots, x_{n}\right\}$ be a finite set of (realvalued) variables. A flow condition $f$ on $V$ is defined by

$$
f::=\dot{x}_{1}=d_{1} \wedge \ldots \wedge \dot{x}_{n}=d_{1}
$$

where $d_{1}, \ldots, d_{n} \in \mathbb{Q}$. We can also write a set of all flow conditions in $F(V)$.

- A finite set Act of actions: An action is either one among input action, output action and internal action. An input action that has the form $m$ ? represents receiving the message $m$. An output action that has the form $m$ ! represents sending (broad-casting) the message $m$. An internal action $a_{\tau}$ represents an asynchronous transition between two locations. In particular, the special actions are below:

- Creation actions: $C r t \_\mathcal{A}_{i}$ ! and $C r t \_\mathcal{A}_{i}$ ? denote the creation of $\mathcal{A}_{i}$.

- Destruction actions: Dst_- $\mathcal{A}_{i}$ ! and $D s t_{-} \mathcal{A}_{i}$ ? denote the destruction of $\overline{\mathcal{A}}_{i}$.

- Enqueue actions: $q_{i} ! m$ denotes enqueueing the message $m$ into the queue $q_{i}$.

- Dequeue actions: $q_{i}$ ? $m$ denotes dequeueing the message $m$ from the queue $q_{i}$. 
- $\quad$ A finite set $T \subseteq L \times \Phi(V) \times A c t \times 2^{U P D(V)} \times L$ of transitions: A constraint of transition $g \in \Phi(V)$ is called guard condition.

$U P D(V)$ is a set of update expressions. An update expression $\lambda$ is defined by

$$
\lambda::=x:=c \mid x:=x+c
$$

where $x \in V$ and $c \in \mathbb{Q}$.

- An initial transition $t_{0} \in L \times\left(A c t_{\text {in }} \cup A c t_{\tau}\right) \times 2^{U P D(V)}$

- A finite set $T_{\text {end }} \subseteq L \times \Phi(V) \times$ Act $_{\text {out }}$ of destruction transitions

2) Semantics of a DLHA: A state $\sigma$ of a DLHA is defined as $(l, \nu)$ or $\perp$, where $l$ is a location, $\nu$ is an evaluation of variables and $\perp$ is an undefined state (that is, the DLHA is not created). An evaluation is an assignment of variables to real numbers.

The operational semantics of a DLHA is defined by the following rules:

- Time transition:

○ For any $d \in \mathbb{R}_{\geq 0}, \perp \Rightarrow_{d} \perp$.

- For $d \in \mathbb{R}_{\geq 0}$ and a state $(l, \nu)$,

$$
(l, \nu) \Rightarrow_{d}(l, \nu+d) \text { if } \nu+d \in \operatorname{Inv}(l)
$$

where $\nu+d$ is a shorthand for a function defined by $\nu(x)+\operatorname{Flow}(x) \cdot d$.

- Discrete transition:

○ For a transition $\left(l, \phi, a, \lambda, l^{\prime}\right) \in T$,

$$
(l, \nu) \Rightarrow_{a}\left(l^{\prime}, \nu[\lambda]\right) \text { if } \nu \in \phi, \nu[\lambda] \in \operatorname{Inv} .
$$

$\circ$ For a destruction-transition $(l, \phi, a) \in T_{\text {end }}$,

$$
(l, \nu) \Rightarrow_{a} \perp \text { if } \nu \in \phi .
$$

○ For the initial transition $t_{0}=\left(l_{0}, a_{0}, \lambda_{0}\right)$,

$$
\perp \Rightarrow_{a_{0}}\left(l_{0}, \overrightarrow{0}[\lambda]\right)
$$

where $\overrightarrow{0}$ is an evaluation that assigns 0 to each variables.

\section{B. Dynamically Reconfigurable System}

A dynamically reconfigurable system consists of a set of DLHAs and a set of queues. Formally, a system $\mathcal{S}$ is defined by a tuple $(A, \mathcal{Q})$, where $A$ is a finite set of DLHAs and $\mathcal{Q}$ is a finite set of queues (unbounded FIfO buffers). A state of the system is a pair $\left\langle\vec{\sigma}, \vec{w}_{\mathcal{Q}}\right\rangle=\left\langle\left(\sigma_{1}, \ldots, \sigma_{|A|}\right),\left(w_{1}, \ldots, w_{|\mathcal{Q}|}\right)\right\rangle$ of a vector of DLHA-states and a vector of queue-contents.

1) Time Transition: For an arbitrary $\delta \in \mathbb{R}_{\geq 0}$, the time transition is defined by the following rule:

$$
\left\langle\vec{\sigma}, \overrightarrow{w_{\mathcal{Q}}}\right\rangle \rightarrow_{\delta}\left(\vec{\sigma}^{\prime}, \vec{w}_{\mathcal{Q}}\right) \Longleftrightarrow \forall i .\left[\sigma_{i} \Rightarrow_{\delta} \sigma_{i}\right]
$$

2) Discrete Transition: Let $\vec{\sigma}, \vec{\sigma}^{\prime}, \vec{w}_{\mathcal{Q}}$ and $\vec{w}_{\mathcal{Q}}^{\prime}$ be

$$
\begin{aligned}
& \vec{\sigma}=\left(\sigma_{1}, \ldots, \sigma_{|A|}\right), \\
& \vec{\sigma}^{\prime}=\left(\sigma_{1}^{\prime}, \ldots, \sigma_{|A|}^{\prime}\right), \\
& \vec{w}_{\mathcal{Q}}=\left(w_{1}, \ldots, w_{|\mathcal{Q}|}\right) \\
& \text { and } \vec{w}_{\mathcal{Q}}^{\prime}=\left(w_{1}^{\prime}, \ldots, w_{|\mathcal{Q}|}^{\prime}\right) .
\end{aligned}
$$

- $\quad$ For any output action $a !,\left\langle\vec{\sigma}, \vec{w}_{\mathcal{Q}}\right\rangle \rightarrow_{a}\left\langle\vec{\sigma}^{\prime}, \vec{w}_{\mathcal{Q}}\right\rangle$

$$
\text { if } \begin{aligned}
\exists i . & {\left[\sigma_{i} \Rightarrow_{a !} \sigma_{i}^{\prime} \wedge \forall j \neq i .\left[\sigma_{j} \Rightarrow_{a ?} \sigma_{j}\right.\right.} \\
\vee & \left.\left.\left(\neg \exists \sigma \cdot\left[\sigma_{j} \Rightarrow_{a ?} \sigma_{j}^{\prime}\right] \wedge \sigma_{j}=\sigma_{j}^{\prime}\right)\right]\right] .
\end{aligned}
$$

An output action is broadcasted to all DLHAs, and a DLHA receiving the action moves by the synchronization if the state holds the guard condition.

- For an internal action $a_{\tau}$,

○ In case of $a_{\tau}=q_{k} ! w,\left\langle\vec{\sigma}, \vec{w}_{\mathcal{Q}}\right\rangle \rightarrow_{q_{k} ! w}$ $\left\langle\vec{\sigma}^{\prime}, \vec{w}_{\mathcal{Q}}^{\prime}\right\rangle$

$$
\text { if } \begin{aligned}
& \exists i . {\left[\sigma_{i} \Rightarrow_{q_{k} ! w} \sigma_{i}^{\prime} \wedge \forall j \neq i .\left[\sigma_{j}=\sigma_{j}^{\prime}\right]\right.} \\
&\left.\wedge w_{k}^{\prime}=w_{k} w \wedge \forall l \neq k .\left[w_{k}=w_{k}^{\prime}\right]\right] .
\end{aligned}
$$

$\circ \quad$ In case of $a_{\tau}=q_{k} ? w,\left\langle\vec{\sigma}, \vec{w}_{\mathcal{Q}}\right\rangle \rightarrow_{q_{k}}$ ?w $\left\langle\vec{\sigma}^{\prime}, \vec{w}_{\mathcal{Q}}^{\prime}\right\rangle$

$$
\text { if } \begin{aligned}
& \exists i {\left[\sigma_{i} \Rightarrow_{q_{k} ? w} \sigma_{i}^{\prime} \wedge \forall j \neq i .\left[\sigma_{j}=\sigma_{j}^{\prime}\right]\right.} \\
&\left.\wedge w_{k}=w w_{k}^{\prime} \wedge \forall l \neq k .\left[w_{l}=w_{l}^{\prime}\right]\right] .
\end{aligned}
$$

○ Otherwise, $\left\langle\vec{\sigma}, \vec{w}_{\mathcal{Q}}\right\rangle \rightarrow_{a_{\tau}}\left\langle\vec{\sigma}^{\prime}, \vec{w}_{\mathcal{Q}}\right\rangle$

$$
\text { if } \exists i .\left[\sigma_{i} \Rightarrow_{a_{\tau}} \sigma_{i}^{\prime} \wedge \forall j \neq i .\left[\sigma_{j}=\sigma_{j}^{\prime}\right]\right] .
$$

A run(or path) $\rho$ of the system $\mathcal{S}$ is the following finite(or infinite) sequence of states.

$$
\rho: s_{0} \rightarrow{ }_{a_{0}}^{\delta_{0}} s_{1} \rightarrow{ }_{a_{1}}^{\delta_{1}} \cdots \rightarrow \rightarrow_{a_{i-1}}^{\delta_{i-1}} s_{i} \rightarrow{ }_{a_{i}}^{\delta_{i}} \cdots
$$

where $\rightarrow{ }_{a_{i}}^{\delta_{i}}$ between $s_{i}$ and $s_{i+1}$ is defined as follows:

$$
s_{i} \rightarrow_{a_{i}}^{\delta_{i}} s_{i+1} \Longleftrightarrow \exists s_{i}^{\prime} \cdot\left[s_{i} \rightarrow_{\delta_{i}} s_{i}^{\prime} \wedge s_{i}^{\prime} \rightarrow_{a_{i}} s_{i+1}\right] .
$$

The initial state $s_{0}$ of a dynamically reconfigurable system is $\left\langle\left(\sigma_{01} \ldots, \sigma_{0|A|}\right),\left(w_{01}, \ldots, w_{0|\mathcal{Q}|}\right)\right\rangle$ where each $\sigma_{0 i}$ is the initial state of DLHA $\mathcal{A}_{i}$ and each $w_{0 j}$ is empty, that is $\forall j . w_{0 j}=\varepsilon$

An example of a dynamically reconfigurable system is shown in Fig.1. This system consists of three DLHAs and one queue.

A DLHA $\mathcal{A}_{1}$ has two locations, Run and Wait, that are expressed as circles. In the location Run, an invariant is $x \leq 10$ and a flow condition is $\dot{x}=1$.

A run of this system is as below:

$$
\begin{aligned}
\rho: & \langle((\text { Run }, x=0),(\text { Idle }, y=0), \perp),(\varepsilon)\rangle \\
& \rightarrow{ }_{q ! \mathcal{A}_{3}}^{10}\left\langle((\text { Wait }, x=10),(\text { Idle }, y=0), \perp),\left(\mathcal{A}_{3}\right)\right\rangle \\
& \rightarrow{ }_{q ? \mathcal{A}_{3}}^{0}\langle((\text { Wait }, x=10),(\text { Create }, y=0), \perp),(\varepsilon)\rangle \\
& \rightarrow{ }_{\text {Crt_- }}^{0}\langle((\text { Wait }, x=10),(\text { Idle }, y=0), \\
& \quad(\text { Execute }, z=0)),(\varepsilon)\rangle \\
& \rightarrow{ }_{\text {Dst_- }}^{50}\langle((\text { Run }, x=0),(\text { Idle }, y=0), \perp),(\varepsilon)\rangle
\end{aligned}
$$


Here, $\varepsilon$ denotes the empty string.

In this tiny system, each DLHA has the following features:

- Environment: $\mathcal{A}_{1}$ periodically sends a message to request creation of $\mathcal{A}_{3}$.

- $\quad$ Dispatcher: $\mathcal{A}_{2}$ receives the message from $\mathcal{A}_{1}$ and creates $\mathcal{A}_{3}$ with actions $C r t \_\mathcal{A}_{3}$ ! and $C r t \_\mathcal{A}_{3}$ ?.

- $\quad$ Task: $\mathcal{A}_{3}$ is created by $\mathcal{A}_{2}$ synchronously with actions Crt_ $\mathcal{A}_{3}$ !.

In the initial state, $\mathcal{A}_{1}$ and $\mathcal{A}_{2}$ is already created because they have initial transitions with internal actions, and the content of the queue is empty (1). Next, $\mathcal{A}_{1}$ sends a creation request of $\mathcal{A}_{3}$ to $\mathcal{A}_{2}$ via the queue (2). Receiving the request asynchronously, $\mathcal{A}_{2}$ prepares to create $\mathcal{A}_{3}$, that is, $\mathcal{A}_{2}$ moves from Idle to Create and the content of the queue becomes empty (3). $\mathcal{A}_{3}$ is created and its state moves from $\perp$ to (Execute, $z=0$ ) (4). When $\mathcal{A}_{3}$ finishes executing the process, it is destroyed with the action Dst_ $\mathcal{A}_{3}$ ! (5).
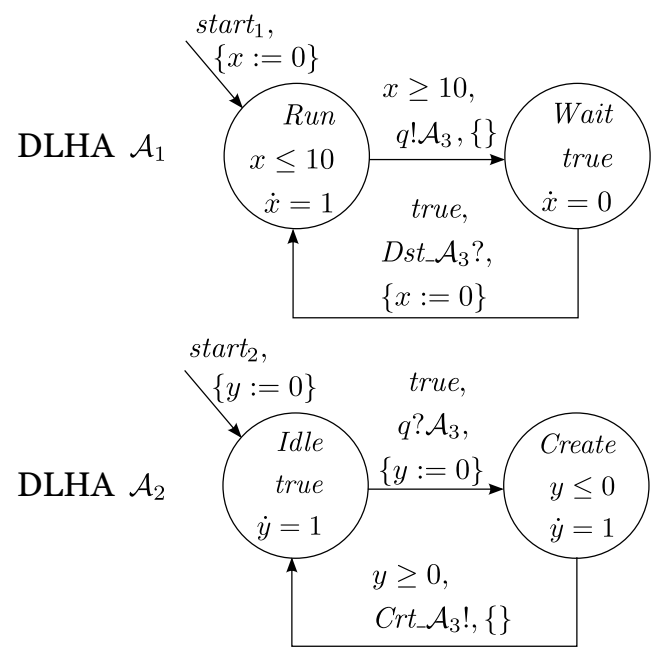

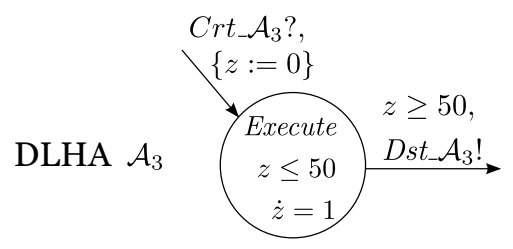

Fig. 1. An example of a dynamically reconfigurable system

\section{REACHABILITY ANALYSIS}

\section{A. Reachability Problem}

Given a dynamically reconfigurable system $\mathcal{S}=(A, Q)$ and a location $l_{t}$, we say " $\mathcal{S}$ reaches $l_{t}$ " if there exists a path from the initial state to a state containing $l_{t}$. The reachability problem is the problem of determining whether $\mathcal{S}$ reaches $l_{t}$.

\section{B. Convex Polyhedra}

In our proposed method, we introduce convex polyhedra for the reachability analysis according to [6]. For a set $V=$ $\left\{x_{1}, \ldots, x_{n}\right\}$ of variables, a convex polyhedron $\zeta$ on $V$ has the following syntax:

$$
\zeta::=\text { true } \mid \text { false }\left|\sum_{i=1}^{n} a_{i} x_{i} \sim a_{n+1}\right| \zeta_{1} \wedge \zeta_{2}
$$

where $\sim \in\{=,<,>, \leq, \geq\}$ and $\zeta_{1}, \zeta_{2}$ are convex polyhedra.

Let $\llbracket \zeta \rrbracket$ be a set of vertices such that are contained in the region described by $\zeta$, that is, $\llbracket \zeta \rrbracket=\exists V$. $[\zeta] \subseteq \mathbb{R}^{n}$.

Then, the equivalence of convex polyhedra is defined as follows:

$$
\zeta_{1}=\zeta_{2} \Longleftrightarrow \llbracket \zeta_{1} \rrbracket=\llbracket \zeta_{2} \rrbracket .
$$

\section{Algorithm}

For the reachability analysis, a state of system is defined as $\left(L, \zeta, \vec{w}_{\mathcal{Q}}\right)$, where $L$ is a set of locations, $\zeta$ is a convex polyhedron, and $\vec{w}_{\mathcal{Q}}$ is a vector of queue-contents. The overview of the reachability analysis is shown in Fig. 2. The analysis is performed by breadth-first order with QDDs[7] as the following procedures:

1) Compute an initial state $s_{0}$ of the system $\mathcal{S}$ (line $1-$ line 3).

2) Initialize a traversed set Visit and a untraversed set Wait of states by $\varnothing$ and $\left\{s_{0}\right\}$ (line 4 ).

3) While Wait is not empty, the following processing is repeated (line 8).

a) Take a state $\left(L, \zeta, \overrightarrow{w_{\mathcal{Q}}}\right)$ from Wait and remove the state from Wait (line 9 - line 10).

b) If the set $L$ of locations contains the target location, return "yes" and terminate (line 11 - line 13).

c) If the state is not traversed yet $\left(\left(L, \zeta, \overrightarrow{w_{\mathcal{Q}}}\right) \notin\right.$ Visit):

i) Add the state into Visit (line 15).

ii) Compute a set $S_{\text {post }}$ of successors by the subroutine Succ (line 17).

iii) Add all components of $S_{\text {post }}$ into Wait (line 18).

The subroutine Succ that computes successors of a state is shown in Fig.3. Given a state $\left(L, \zeta, \vec{w}_{\mathcal{Q}}\right)$ and a system, successors are computed by the following procedure:

1) For each transition $\left(l, \phi, a, \lambda, l^{\prime}\right)$ (or destructiontransition $\left.\left(l, \phi, a_{l} !\right)\right)$ outgoing from a location $l \in L$, a set $S_{\text {post }}$ of post states is computed as below:

2) If $a$ is an enqueue action $q_{k} ! w$ : Add a message $w$ to the content of queue $q_{k}$ and add the new state to $S_{\text {post }}$ (line 11 - line 15).

3) If $a$ is a dequeue action $q_{k} ? w$ : Remove a message $w$ from the content of queue $q_{k}$ and add the new state to $S_{\text {post }}$ (line 16 - line 19).

4) If $a$ is other internal action: Add the new state to $S_{\text {post }}$ (line 22).

5) If $a$ is an output action $a_{l} !: S_{\text {post }}$ is computed by subroutine Syncs as the following steps (line 29).

a) Compute a set $T_{\text {sync }}$ of transitions synchronizing with $t$.

b) Compute a set $L^{\prime}$ of locations of the new state with $t$ and $T_{\text {sync }}$. 
Input: a system $\mathcal{S}$ and a target location $l_{t}$

Output: "yes" or "no"

1: $/ *$ Compute the initial state */

2: $L_{0} \leftarrow\left\{l_{0 i} \mid t_{0 i}=\left(l_{0 i}, a_{0 i}, \lambda_{0 i}\right), a_{0 i} \neq C r t \mathcal{A}_{i} ?\right\}$

3: $\lambda_{0} \leftarrow \bigcup\left\{\lambda_{0 i} \mid t_{0 i}=\left(l_{0 i}, a_{0 i}, \lambda_{0 i}\right), a_{0 i} \neq \bar{C} r t \mathcal{A}_{i} ?\right\}$

4: $s_{0} \leftarrow\left(L_{0}, \overrightarrow{0}\left[\lambda_{0}\right],(\varepsilon, \ldots, \varepsilon)\right)$

5: $\quad / *$ Initialize a set Visit of visited states and a queue Wait of states to search */

: Visit $\leftarrow \varnothing$, Wait $\leftarrow\left\{s_{0}\right\}$

/* Traverse in a breadth-first order $* /$

while Wait $\neq \varnothing$ do

$\left(L, \zeta, \vec{w}_{\mathcal{Q}}\right) \leftarrow s \in$ Wait

Wait $\leftarrow$ Wait $\backslash\left\{\left(L, \zeta, \vec{w}_{\mathcal{Q}}\right)\right\}$

if $l_{t} \in L$ then return "yes"

if $\left(L, \zeta, \vec{w}_{\mathcal{Q}}\right) \notin$ Visit then

Visit $\leftarrow$ Visit $\cup\left\{\left(L, \zeta, \vec{w}_{\mathcal{Q}}\right)\right\}$

/* Compute a set of post-states $* /$

$S_{\text {post }} \leftarrow \operatorname{Succ}\left(\left(L, \zeta, \vec{w}_{\mathcal{Q}}\right), \mathcal{S}\right)$

Wait $\leftarrow$ Wait $\cup S_{\text {post }}$

return "no"

Fig. 2. Algorithm of Reachability Analysis

c) Compute a convex polyhedron $\zeta^{\prime}$ of the new state with $t$ and $T_{\text {sync }}$.

d) Add the new state $\left(L^{\prime}, \zeta^{\prime}, w_{\mathcal{Q}}\right)$ to $S_{\text {post }}$.

6) If $a$ is an input action: $S_{\text {post }}=\emptyset$.

\section{IMPLEMENTATION AND PRACTICAL EXPERIMENTS}

We have developed a prototype model checker of dynamically reconfigurable systems. The model checker is implemented in Java and comprised of about 1,600 lines of code using external libraries LAS[8], PPL[9], and QDD[7], [10].

Moreover, for practical experiments, we have specified a cooperating system consisting CPU and DRP(Dynamically Reconfigurable Processor), and verified several safety properties for the system. The configuration of the embedded system is shown in Fig.4, and components of the system is shown in Fig.5. This system consists of 11 DLHAs and 1 queue. We show the DLHA of Scheduler in Fig.6 for example. The external environment consists of EnvA and EnvB that create periodically TaskA and TaskB. That is, EnvA creates TaskA with Crt_taskA! every 70 milliseconds and EnvB creates TaskB with Crt_taskB! every 200 milliseconds. Scheduler performs scheduling in accordance with the priority and actions for creation and destruction of DLHAs. For example, when TaskA is created by EnvA with Crt_taskA! and TaskB is already running, Scheduler receives Crt_taskA? and sends Act_Preempt!. Then, TaskA moves to location RunA and TaskB moves to the location WaitB with the action.

TaskA and TaskB send a message to Sender if they need a co-task. Sender enqueues the message to create a cotask to the queue $q$ when it receives a message from tasks. When TaskA moves to location RunA from location WaitA with Act_Create_a0!, Sender receives Act_Create_a0? and enqueues cotask_a0 to $q$ with $q$ ! cotask_a0.

DRP_Dispatcher dequeues a message and creates cotask_a0, cotask_a1 and cotask_b0 if there are enough free tiles. Frequency_Manager is a module which manages the operating frequency of DRP. When a DLHA of co-task is created, Frequency_Manager moves to the location fixing the frequency to minimum value.

For example, we have verified the schedulability of tasks. This property provides that each task must be finished processing within its deadline. To verify the property, a monitor automaton is defined as Fig.7. Monitor automaton checks whether the system satisfies the property, and it moves to a special location called error location when the property is not satisfied. In this case, the schedulability is verified in 180 seconds with $169[\mathrm{MB}]$ on a machine with Intel (R) Core (TM) i7-3770 (3.40 [GHz]) CPU and 16 [GB] RAM.

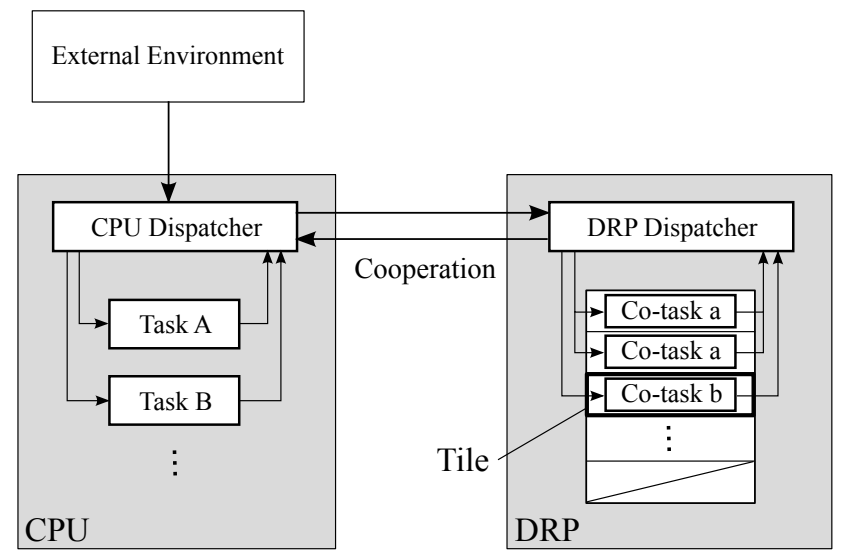

Fig. 4. Overview of the CPU-DRP embedded system

\section{CONCLUSION}

In this paper, we have presented the specification language of dynamically reconfigurable systems and the reachability analysis algorithm for the verification. The next step would be to focus on more effective methods (e.g., Counterexample Guided Abstraction Refinement, Satisfiability Modulo Theories, etc.) for the verification. 
Input: a state $\left(L, \zeta, \vec{w}_{\mathcal{Q}}\right)$ and the system $\mathcal{S}$

Output: the set $S_{\text {post }}$ of post-states

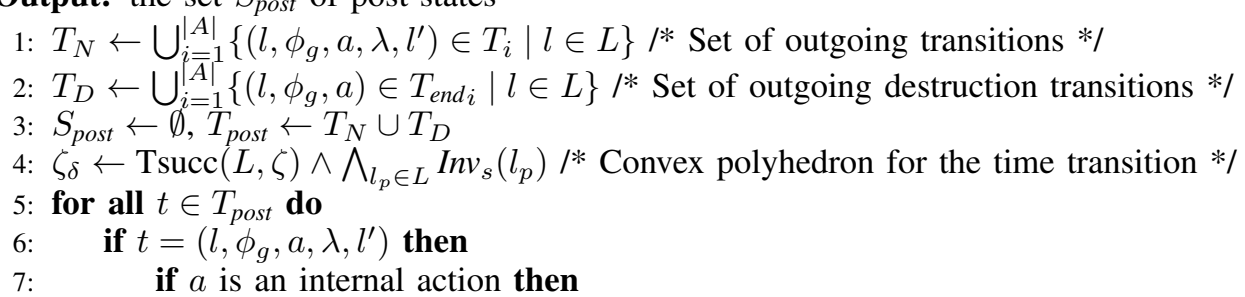

$$
\begin{aligned}
& L^{\prime} \leftarrow(L \backslash\{l\}) \cup\left\{l^{\prime}\right\} / * \text { Locations of the post-state */ } \\
& \zeta^{\prime} \leftarrow\left(\zeta_{\delta} \wedge \phi_{g}\right)[\lambda] \wedge \zeta_{i}^{\prime} \wedge \bigwedge_{l_{p}^{\prime} \in L^{\prime}} \operatorname{Inv} v_{s}\left(l_{p}^{\prime}\right) / * \text { The convex polyhedron of the post-states */ } \\
& \text { if } \zeta^{\prime} \neq \text { false then } \\
& \quad \text { if } a \text { is a enqueue action } q_{k} ! w \text { then }
\end{aligned}
$$$$
\left(w_{1}, \ldots, w_{|\mathcal{Q}|}\right) \leftarrow \vec{w}_{\mathcal{Q}}
$$$$
w_{k}^{\prime} \leftarrow w_{k} w / * \text { Enqueue the message into } q_{k} * /
$$$$
\vec{w}_{\mathcal{Q}}^{\prime} \leftarrow\left(w_{1}, \ldots, w_{k-1}, w_{k}^{\prime}, w_{k+1}, \ldots, w_{|\mathcal{Q}|}\right)
$$$$
S_{\text {post }}^{\mathcal{Q}} \leftarrow S_{\text {post }} \cup\left\{\left(L^{\prime}, \zeta^{\prime}, \vec{w}_{\mathcal{Q}}^{\prime}\right)\right\}
$$

else if $a$ is a dequeue action $q_{k}$ ? $w$ then

$$
\begin{aligned}
\text { if } w_{k} & =w w_{k}^{\prime} \text { then } \\
\vec{w}_{\mathcal{Q}}^{\prime} & \leftarrow\left(w_{1}, \ldots, w_{k-1}, w_{k}^{\prime}, w_{k+1}, \ldots, w_{|\mathcal{Q}|}\right) / * \text { Dequeue the message from } q_{k} * /
\end{aligned}
$$$$
S_{\text {post }} \leftarrow S_{\text {post }} \cup\left\{\left(L^{\prime}, \zeta^{\prime}, \vec{w}_{\mathcal{Q}}^{\prime}\right)\right\}
$$

else

$$
S_{\text {post }} \leftarrow S_{\text {post }} \cup\left\{\left(L^{\prime}, \zeta^{\prime}, \vec{w}_{\mathcal{Q}}\right)\right\} / * \text { For other internal action */ }
$$

else if $a$ is an output action $a_{l}$ ! then

$$
S_{\text {post }} \leftarrow S_{\text {post }} \cup \operatorname{Syncs}\left(\left(L, \zeta, \vec{w}_{\mathcal{Q}}\right), t, \mathcal{S}\right) / * \text { Compute the set of states by the synchronous transitions */ }
$$

else

25: $\quad S_{\text {post }} \leftarrow S_{\text {post }} \cup \operatorname{Syncs}\left(\left(L, \zeta, \vec{w}_{\mathcal{Q}}\right), t, \mathcal{S}\right) / *$ Compute the set of states by the destruction transition */

Fig. 3. Subroutine Succ

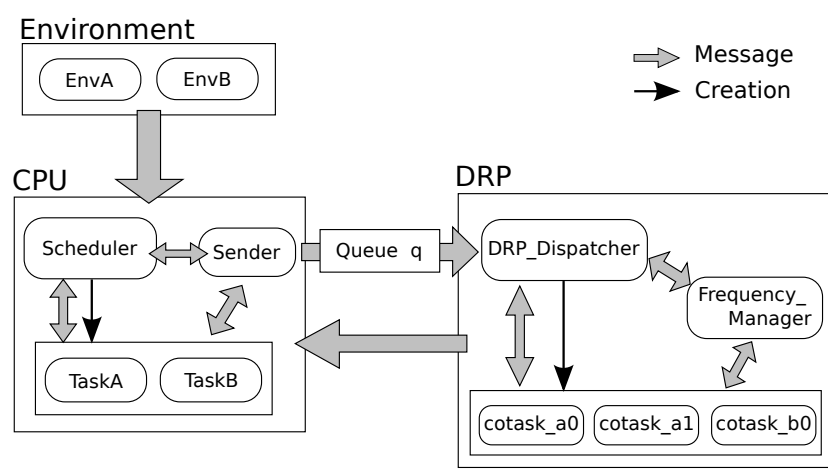

Fig. 5. Components of the system

\section{REFERENCES}

[1] R. Alur, C. Courcoubetis, T. A. Henzinger, and P. H. Ho, "Hybrid automata: An algorithmic approach to the specification and verification of hybrid systems," Lecture Notes in Computer Science, vol. 736, pp. 209-229, 1993.

[2] S. Minami, S. Takinai, S. Sekoguchi, Y. Nakai, and S. Yamane, "Modeling, specification and model checking of dynamically reconfigurable processors," in Computer Software 28(1). Japan Society for Software Science and Technology, 2011, pp. 190-216.

[3] P. C. Attie and N. A. Lynch, "Dynamic input/output automata, a formal model for dynamic systems," in Proceedings of the twentieth annual ACM symposium on Principles of distributed computing, ser. PODC '01, 2001, pp. 314-316.

[4] H. Yamada, Y. Nakai, and S. Yamane, "Proposal of specification language and verification experiment for dynamically reconfigurable

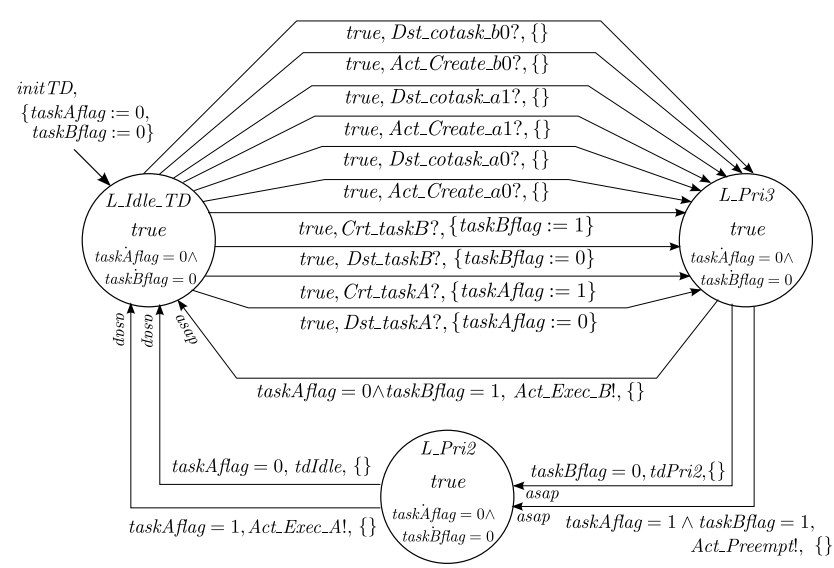

Fig. 6. DLHA of Scheduler

system," Journal of Information Processing Society of Japan, Programming, vol. 6, no. 3, pp. 1-19, 2013.

[5] H. Nakano, T. Shindo, T. Kazami, and M. Motomura, "Development of dynamically reconfigurable processor LSI," NEC Technical Journal, vol. 56, no. 2, pp. 99-102, 2003.

[6] R. Alur, C. Courcoubetis, N. Halbwachs, T. A. Henzinger, P.-H. Ho, X. Nicollin, A. Olivero, J. Sifakis, and S. Yovine, "The algorithmic analysis of hybrid systems," THEORETICAL COMPUTER SCIENCE, vol. 138, pp. 3-34, 1995.

[7] B. Boigelot and P. Godefroid, "Symbolic verification of communication protocols with infinite statespaces using qdds," Form. Methods Syst. Des., vol. 14, no. 3, pp. 237-255, 1999. 


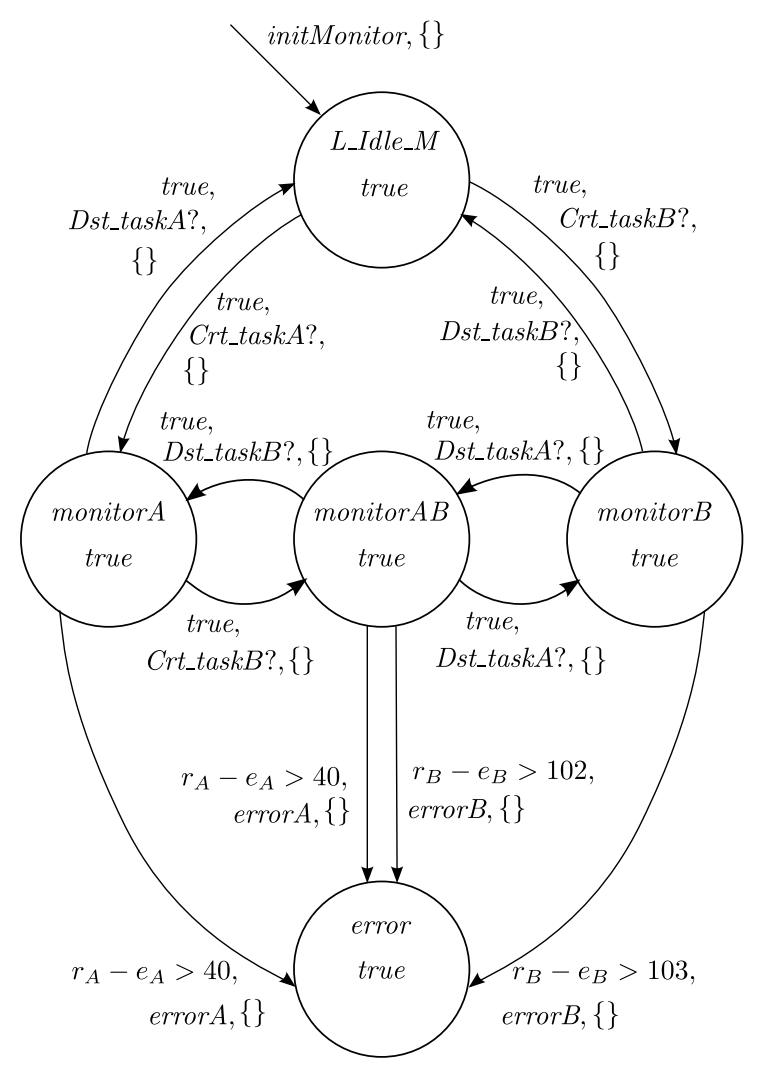

Fig. 7. Monitor automaton for schedulability

[8] Y. Ono and S. Yamane, "Computation of quantifier elimination of linear inequlities of first order predicate logic," IEICE Technical Report. COMP, Computation, vol. 111, no. 20, pp. 55-59, 2011.

[9] R. Bagnara, P. M. Hill, and E. Zaffanella, "The parma polyhedra library: Toward a complete set of numerical abstractions for the analysis and verification of hardware and software systems," Sci. Comput. Program., vol. 72, no. 1-2, pp. 3-21, 2008.

[10] B. Boigelot, P. Godefroid, B. Willems, and P. Wolper, "The power of qdds (extended abstract)," in SAS, 1997, pp. 172-186. 